POS $\quad$ PROCEEDINGS

\title{
Heavy Majorana neutrino effects on MSSM-Mh
}

\author{
Maria Herrero*t \\ Instituto de Fisica Teorica, UAM/CSIC, Madrid, Spain \\ E-mail: maria.herrero@uam.es
}

\section{Sven Heinemeyer}

Instituto de Fisica de Cantabria, IFCA-CSIC, Santander, Spain

E-mail: sven.heinemeyerecern.ch

\section{Siannah Peñaranda}

Departamento de Fisica Teorica, Universidad de Zaragoza, Spain

E-mail: siannaheunizar.es

\section{Ana M. Rodriguez-Sanchez}

Instituto de Fisica Teorica, UAM/CSIC, Madrid, Spain

E-mail: anam.rodriguez@uam.es

\begin{abstract}
We study the effects of heavy Majorana neutrinos on the Higgs sector of the MSSM via radiative corrections. We work within the SUSY context where the MSSM particle content is enlarged with right handed neutrinos and their corresponding SUSY partners, the sneutrinos, and where compatibility with neutrino data is required. We compute the one-loop corrections to the mass of the lightest MSSM CP-even neutral Higgs boson from Majorana neutrinos and their SUSY partners and assume a seesaw mechanism of type I for neutrino mass generation. A negative and sizeable Higgs mass correction of up to $-5 \mathrm{GeV}$ is found for a heavy Majorana mass of up to $10^{15}$ $\mathrm{GeV}$. This negative correction can grow up to several tens of GeV if the soft SUSY breaking mass associated to their sneutrino partners is simmilarly heavy as the Majorana mass.
\end{abstract}

10th International Symposium on Radiative Corrections (Applications of Quantum Field Theory to Phenomenology) - Radcor2011

September 26-30, 2011

Mamallapuram, India

\footnotetext{
*Speaker.

${ }^{\dagger}$ Talk given at RADCOR2011, September 26-30,2011, Mamallapuram, India.
} 


\section{Introduction}

The current experimental data on neutrino mass differences and neutrino mixing angles clearly indicate new physics beyond the so far successful Standard Model of Particle Physics (SM). In particular, neutrino oscillations imply that at least two generations of neutrinos must be massive. Therefore, one needs to extend the SM to incorporate neutrino mass terms.

We assume here the simplest version of a SUSY extension of the SM, the well known Minimal Supersymmetric Standard Model (MSSM), extended by right-handed Majorana neutrinos and their SUSY partners, and where the seesaw mechanism of type I [1] is implemented to generate the small neutrino masses. For simplicity, we focus here in the one generation case which already provides interesting results and allows for a clearer illustration of the most relevant behaviour of the radiative corrections with the Majorana mass scale. The three generation case will be postponed for a later work.

On the other hand, it is well known that heavy Majorana neutrinos, with $m_{M} \sim 10^{13}-10^{15}$ $\mathrm{GeV}$, induce large LFV rates [2], due to their potentially large Yukawa couplings to the Higgs sector. For the same reason, radiative corrections to Higgs boson masses due to such heavy Majorana neutrinos could also be relevant. Consequently, our study has been focused on the radiative corrections to the lightest MSSM CP-even $h$ boson mass, $M_{h}$, due to the one-loop contributions from the neutrino/sneutrino sector within the MSSM-seesaw framework.

In the following we briefly review the main relevant aspects of the calculation of the mass corrections and the numerical results. For further details we address the reader to the full version of our work [3], where also an extensive list with references to related works can be found.

\section{The basics of the MSSM-seesaw model}

The MSSM-seesaw model with one neutrino/sneutrino generation is described in terms of the well known MSSM superpotential plus the new relevant terms contained in:

$$
W=\varepsilon_{i j}\left[Y_{v} \hat{H}_{2}^{i} \hat{L}^{j} \hat{N}-Y_{l} \hat{H}_{1}^{i} \hat{L}^{j} \hat{R}\right]+\frac{1}{2} \hat{N} m_{M} \hat{N}
$$

where $m_{M}$ is the Majorana mass and $\hat{N}=\left(\tilde{v}_{R}^{*},\left(v_{R}\right)^{c}\right)$ is the additional superfield that contains the right-handed neutrino $v_{R}$ and its scalar partner $\tilde{v}_{R}$.

There are also new relevant terms in the soft SUSY breaking potential:

$$
V_{\text {soft }}^{\tilde{v}}=m_{\tilde{L}}^{2} \tilde{v}_{L}^{*} \tilde{v}_{L}+m_{\tilde{R}}^{2} \tilde{\tilde{V}}_{R}^{*} \tilde{V}_{R}+\left(Y_{v} A_{v} H_{2}^{2} \tilde{v}_{L} \tilde{v}_{R}^{*}+m_{M} B_{v} \tilde{v}_{R} \tilde{v}_{R}+\text { h.c. }\right) \text {. }
$$

After electro-weak (EW) symmetry breaking, the charged lepton and Dirac neutrino masses can be written as

$$
m_{l}=Y_{l} v_{1}, \quad m_{D}=Y_{v} v_{2},
$$

where $v_{i}$ are the vacuum expectation values (VEVs) of the neutral Higgs scalars, with $v_{1(2)}=$ $v \cos (\sin ) \beta$ and $v=174 \mathrm{GeV}$.

The $2 \times 2$ neutrino mass matrix is given in terms of $m_{D}$ and $m_{M}$ by:

$$
M^{v}=\left(\begin{array}{cc}
0 & m_{D} \\
m_{D} & m_{M}
\end{array}\right) \text {. }
$$


Diagonalization of $M^{v}$ leads to two mass eigenstates, $n_{i}(i=1,2)$, which are Majorana fermions with the respective mass eigenvalues given by:

$$
m_{v, N}=\frac{1}{2}\left(m_{M} \mp \sqrt{m_{M}^{2}+4 m_{D}^{2}}\right) .
$$

The mixing angle that defines the mass eigenstates is given by,

$$
\tan \theta=-\frac{m_{v}}{m_{D}}=\frac{m_{D}}{m_{N}} .
$$

In the seesaw limit, i.e. when $\xi \equiv \frac{m_{D}}{m_{M}} \ll 1$ :

$$
m_{v}=-m_{D} \xi+\mathscr{O}\left(m_{D} \xi^{3}\right) \simeq-\frac{m_{D}^{2}}{m_{M}}, \quad m_{N}=m_{M}+\mathscr{O}\left(m_{D} \xi\right) \simeq m_{M}
$$

and $\theta$ is small, therefore, $v$ is made predominantly of $v_{L}$ and its c-conjugate, $\left(v_{L}\right)^{c}$, whereas $N$ is made predominantly of $v_{R}$ and its c-conjugate, $\left(v_{R}\right)^{c}$.

Regarding the sneutrino sector, the sneutrino mass matrices for the CP-even, $\tilde{M}_{+}$, and the CP-odd, $\tilde{M}_{-}$, subsectors are given respectively by

$$
\tilde{M}_{ \pm}^{2}=\left(\begin{array}{cc}
m_{\tilde{L}}^{2}+m_{D}^{2}+\frac{1}{2} M_{Z}^{2} \cos 2 \beta & m_{D}\left(A_{v}-\mu \cot \beta \pm m_{M}\right) \\
m_{D}\left(A_{v}-\mu \cot \beta \pm m_{M}\right) & m_{\tilde{R}}^{2}+m_{D}^{2}+m_{M}^{2} \pm 2 B_{v} m_{M}
\end{array}\right) .
$$

The diagonalization of these two matrices, $\tilde{M}_{ \pm}^{2}$, leads to four sneutrino mass eigenstates, $\tilde{n}_{i}(i=$ $1,2,3,4)$. In the seesaw limit, where $m_{M}$ is much bigger than all the other mass scales the corresponding sneutrino masses are given by:

$$
\begin{aligned}
m_{\tilde{v}_{+}, \tilde{v}_{-}}^{2} & =m_{\tilde{L}}^{2}+\frac{1}{2} M_{Z}^{2} \cos 2 \beta \mp 2 m_{D}\left(A_{v}-\mu \cot \beta-B_{v}\right) \xi, \\
m_{\tilde{N}_{+}, \tilde{N}_{-}}^{2} & =m_{M}^{2} \pm 2 B_{v} m_{M}+m_{\tilde{R}^{2}}^{2}+2 m_{D}^{2} .
\end{aligned}
$$

The mixing angles defining the sneutrinos mass eigenstates, $\theta_{ \pm}$, are small in this limit and, therefore, $\tilde{v}_{+}$and $\tilde{v}_{-}$are made predominantly of $\tilde{v}_{L}$ and its c-conjugate, $\tilde{v}_{L}^{*}$, whereas $\tilde{N}_{+}$and $\tilde{N}_{-}$are made predominantly of $\tilde{v}_{R}$ and its c-conjugate, $\tilde{v}_{R}^{*}$.

Regarding the relevant interactions for the present work, there are pure gauge interactions between the left-handed neutrinos and the $Z$ boson, those between the 'left-handed' sneutrinos and the Higgs bosons, and those between the 'left-handed' sneutrinos and the $Z$ bosons. All of these are common to the MSSM. In addition, in this MSSM-seesaw scenario, there are interactions driven by the neutrino Yukawa couplings (or equivalently $m_{D}$ since $Y_{v}=\left(g m_{D}\right) /\left(\sqrt{2} M_{W} \sin \beta\right)$ ), as for instance $g_{h v_{L} v_{R}}=-\frac{i g m_{D} \cos \alpha}{2 M_{W} \sin \beta}$, and new interactions due to the Majorana nature driven by $m_{M}$ which are not present in the case of Dirac fermions, as for instance $g_{h \tilde{v}_{L} \tilde{v}_{R}}^{\prime}=-\frac{i g m_{D} m_{M} \cos \alpha}{2 M_{W} \sin \beta}$.

Finally, concerning the size of the new parameters that have been introduced in this model, in addition to those of the MSSM, i.e., $m_{M}, m_{D}, m_{\tilde{R}}, A_{v}$ and $B_{v}$, there are no significant constraints. In the literature it is often assumed that $m_{M}$ has a very large value, $m_{M} \sim \mathscr{O}\left(10^{14-15}\right) \mathrm{GeV}$, in order to get small physical neutrino masses $\left|m_{v}\right| \sim 0.1-1 \mathrm{eV}$ with large Yukawa couplings $Y_{v} \sim \mathscr{O}(1)$. This is an interesting possibility since it can lead to important phenomenological implications due to the large size of the radiative corrections driven by these large Yukawa couplings. We have explored, however, not only these extreme values but the full range for $m_{M}$ from the electroweak scale $\sim 10^{2} \mathrm{GeV}$ up to $\sim 10^{15} \mathrm{GeV}$. 


\section{The basics of the calculation}

In the Feynman diagrammatic (FD) approach the higher-order corrected CP-even Higgs boson masses in the MSSM, denoted here as $M_{h}$ and $M_{H}$, are derived by finding the poles of the $(h, H)$ propagator matrix, which is equivalent to solving the following equation [4]:

$$
\left[p^{2}-m_{h}^{2}+\hat{\Sigma}_{h h}\left(p^{2}\right)\right]\left[p^{2}-m_{H}^{2}+\hat{\Sigma}_{H H}\left(p^{2}\right)\right]-\left[\hat{\Sigma}_{h H}\left(p^{2}\right)\right]^{2}=0 .
$$

where $m_{h, H}$ are the tree level masses. The one loop renormalized self-energies, $\hat{\Sigma}_{\phi \phi}\left(p^{2}\right)$, in (10) can be expressed in terms of the bare self-energies, $\Sigma_{\phi \phi}\left(p^{2}\right)$, the field renormalization constants $\delta Z_{\phi \phi}$ and the mass counter terms $\delta m_{\phi}^{2}$, where $\phi$ stands for $h, H$. For example, the lightest Higgs boson renormalized self energy reads:

$$
\hat{\Sigma}_{h h}\left(p^{2}\right)=\Sigma_{h h}\left(p^{2}\right)+\delta Z_{h h}\left(p^{2}-m_{h}^{2}\right)-\delta m_{h}^{2},
$$

Regarding the renormalization prescription, we have used an on-shell renormalization scheme for $M_{Z}, M_{W}$ and $M_{A}$ mass counterterms and $T_{h}, T_{H}$ tadpole counterterms. On the other hand, we have used a modified $\overline{\mathrm{DR}}$ scheme $(\mathrm{m} \overline{\mathrm{DR}})$ for the renormalization of the wave function and $\tan \beta$. The $\mathrm{m} \overline{\mathrm{DR}}$ scheme is very similar to the well known $\overline{\mathrm{DR}}$ scheme but instead of subtracting the usual $\Delta=\frac{2}{\varepsilon}-\gamma_{E}+\log (4 \pi)$ one subtracts $\Delta_{m}=\Delta-\log \left(m_{M}^{2} / \mu_{\overline{\mathrm{DR}}}^{2}\right)$, hence, avoiding large logarithms of the large scale $m_{M}$. As studied in other works [5], this scheme minimizes higher order corrections when two very different scales are involved in a calculation of radiative corrections.

Since we are interested in exploring the relevance of the new radiative corrections to the lightest Higgs mass from the neutrino/sneutrino sector, we will present here our results in terms of the mass difference with respect to the MSSM prediction. Consequently, we define,

$$
\Delta m_{h}^{\mathrm{m} \overline{\mathrm{DR}}}:=M_{h}^{v / \tilde{v}}-M_{h},
$$

where $M_{h}^{v / \tilde{v}}$ denotes the pole for the light Higgs mass including the $v / \tilde{v}$ corrections (i.e. in the MSSM-seesaw model), and $M_{h}$ the corresponding pole in the MSSM, i.e without the $v / \tilde{v}$ corrections. Thus, for a given set of input parameters we first calculate $M_{h}$ in the MSSM with the help of FeynHiggs [4], such that all relevant known higher-order corrections are included, and then we compute $\Delta m_{h}^{\mathrm{m} \overline{\mathrm{DR}}}$. The full one-loop neutrino/sneutrino corrections to the self-energies, $\hat{\Sigma}_{h h}^{v / \tilde{v}}, \hat{\Sigma}_{H H}^{v / \tilde{v}}$ and $\hat{\Sigma}_{h H}^{v / \tilde{v}}$, entering in the evaluation of $\Delta m_{h}^{\mathrm{m}} \overline{\mathrm{DR}}$ have been evaluated with the help of FeynArts [6] and Form Calc [7].

\section{Analytical and Numerical Results}

We have fully explored the dependence with all the new parameters in [3] and found that the radiative corrections to the Higgs mass are mostly sensitive to $m_{M}, m_{v}, B_{v}$ and $m_{\tilde{R}}$. We focus here on $m_{M}, m_{v}$ and $m_{\tilde{R}}$ leading to the largest corrections and address the reader to [3] for the complete study in terms of all the parameters.

First, in order to compare systematically our predictions of the neutrino/sneutrino sector in the MSSM-seesaw with those in the MSSM, we have split the full one-loop neutrino/sneutrino result into two parts:

$$
\left.\hat{\Sigma}\left(p^{2}\right)\right|_{\text {full }}=\left.\hat{\Sigma}\left(p^{2}\right)\right|_{\text {gauge }}+\left.\hat{\Sigma}\left(p^{2}\right)\right|_{\text {Yukawa }}
$$


where $\left.\hat{\Sigma}\left(p^{2}\right)\right|_{\text {gauge }}$ means the contributions from pure gauge interactions and they are obtained by switching off the Yukawa interactions, i.e. by setting $Y_{V}=0$ (or equivalently $m_{D}=0$ ). The remaining part is named here $\left.\hat{\Sigma}\left(p^{2}\right)\right|_{\text {Yukawa }}$ and refers to the contributions that are only present if $Y_{v} \neq 0$. In other words, this separation splits the full result into the common part with the MSSM, given by $\left.\hat{\Sigma}\left(p^{2}\right)\right|_{\text {gauge }}$, and the new contributions due to the presence of Majorana neutrinos with non vanishing Yukawa interactions, given by $\left.\hat{\Sigma}\left(p^{2}\right)\right|_{\text {Yukawa. }}$ Thus, by comparing the size of these two parts, within the allowed parameter space region, we will localize the areas where $\left.\left.\hat{\Sigma}\left(p^{2}\right)\right|_{\text {Yukawa }} \gg \hat{\Sigma}\left(p^{2}\right)\right|_{\text {gauge }}$, which will therefore indicate a significant departure from the MSSM result. We see in Fig. 1 that the Yukawa part clearly dominates for large $m_{M} \sim 10^{14-15} \mathrm{GeV}$ and
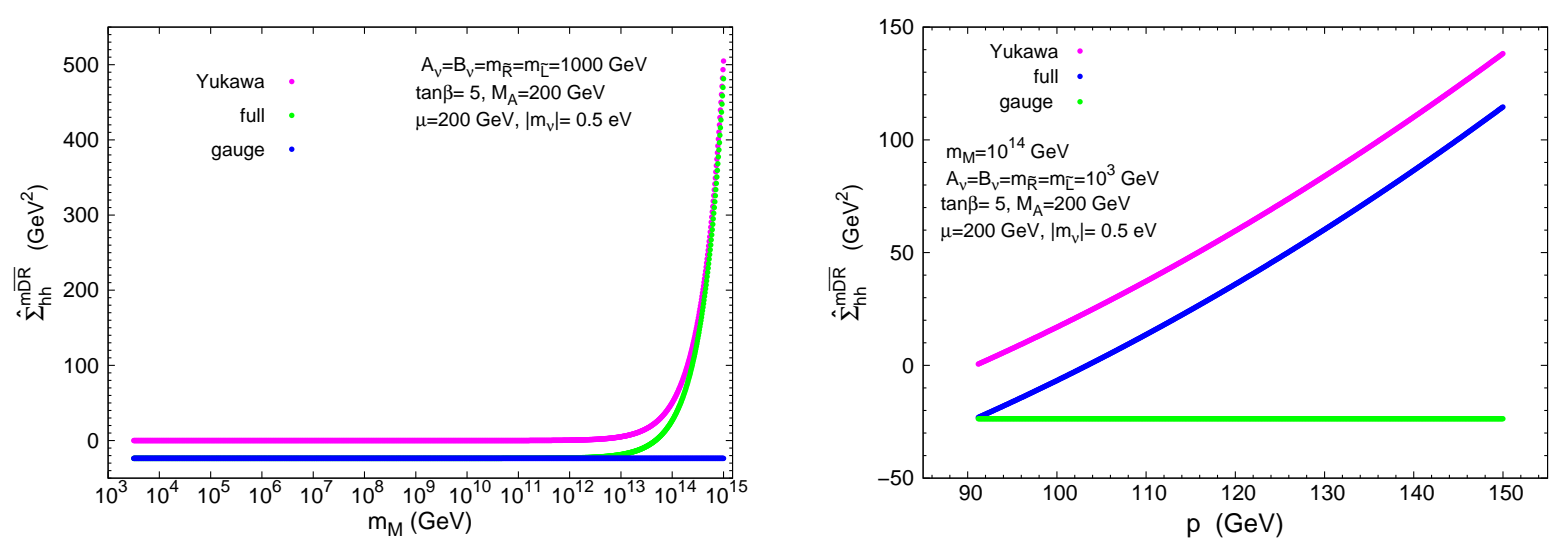

Figure 1: Left panel: Renormalized Higgs boson self-energy as a function of $m_{M}$. Here, $p=116 \mathrm{GeV}$. Right panel: Renormalized Higgs boson self-energy as a function of the external momentum $p$. In both panels, the two contributions from the gauge and Yukawa parts and the full result are shown separately.

that it shows a relevant external momentum dependence. Therefore, at large $m_{M}$, to keep just the Yukawa part is a good approximation, but to neglect the momentum dependence or to set the external momentum to zero are certainly not. In consequence, the alternative effective potential method will not provide a realistic result for the radiative corrections to the Higgs mass.

Second, in order to understand in simple terms the analytical behavior of our full numerical results we have expanded the renormalized self-energies in powers of the seesaw parameter $\xi=$ $m_{D} / m_{M}$ :

$$
\hat{\Sigma}\left(p^{2}\right)=\left(\hat{\Sigma}\left(p^{2}\right)\right)_{m_{D}^{0}}+\left(\hat{\Sigma}\left(p^{2}\right)\right)_{m_{D}^{2}}+\left(\hat{\Sigma}\left(p^{2}\right)\right)_{m_{D}^{4}}+\ldots
$$

The zeroth order of this expansion corresponds to the gauge contribution and it does not depend on $m_{D}$ or $m_{M}$. The rest of the expansion corresponds to the Yukawa contribution. The leading term of this Yukawa contribution is the $\mathscr{O}\left(m_{D}^{2}\right)$ term, because it is the only one not suppressed by the Majorana scale. In fact it goes as $Y_{v}^{2} M_{\mathrm{EW}}^{2}$, where $M_{\mathrm{EW}}^{2}$ denotes generically the electroweak scales involved, concretely, $p^{2}, M_{Z}^{2}$ and $M_{A}^{2}$. In particular, the $\mathscr{O}\left(p^{2} m_{D}^{2}\right)$ terms of the renormalized self-energy, which turn out to be among the most relevant leading contributions, separated into the neutrino and sneutrino contributions, are the following: 


$$
\left.\hat{\Sigma}_{h h}^{\mathrm{m} \overline{\mathrm{DR}}}\right|_{m_{D}^{2} p^{2}} \sim h--\underbrace{v_{L}}_{v_{R}}-h+h-\left.h\right|_{m_{D}^{2} p^{2}} ^{\tilde{v}_{L}} \sim \frac{g^{2} p^{2} m_{D}^{2} c_{\alpha}^{2}}{64 \pi^{2} M_{W}^{2} s_{\beta}^{2}}+\frac{g^{2} p^{2} m_{D}^{2} c_{\alpha}^{2}}{64 \pi^{2} M_{W}^{2} s_{\beta}^{2}}
$$

Notice that the above neutrino contributions come from the Yukawa interaction $g_{h v_{L} v_{R}}$, which is extremely suppressed in the Dirac case but can be large in the present Majorana case. On the other hand, the above sneutrino contributions come from the new couplings $g_{h \tilde{V}_{L} \tilde{V}_{R}}^{\prime}$, which are not present in the Dirac case. It is also interesting to remark that these terms, being $\sim p^{2}$ are absent in both the effective potential and the RGE approaches.

An useful formula for the Higgs mass correction has then be derived, by keeping just the dominant $\mathscr{O}\left(m_{D}^{2}\right)$ contribution to the Yukawa part of the renormalized self-energy:

$$
\begin{aligned}
\Delta m_{h}^{\mathrm{m} \overline{\mathrm{DR}}} & \simeq-\frac{\hat{\Sigma}_{h h}^{v / \tilde{v}}\left(M_{h}^{2}\right)}{2 M_{h}} \approx-\frac{\left(\hat{\Sigma}_{h h}^{\mathrm{mDR}}\left(M_{h}^{2}\right)\right)_{m_{D}^{2}}}{2 M_{h}} \approx \\
& \frac{-g^{2} m_{D}^{2}}{128 \pi^{2} M_{W}^{2} M_{h} \sin ^{2} \beta}\left[-2 M_{A}^{2} \cos ^{2}(\alpha-\beta) \cos ^{2} \beta+2 M_{h}^{2} \cos ^{2} \alpha\right. \\
& \left.-M_{Z}^{2} \sin \beta \sin (\alpha+\beta)\left(2\left(1+\cos ^{2} \beta\right) \cos \alpha-\sin 2 \beta \sin \alpha\right)\right] .
\end{aligned}
$$

We have checked that this simple expression reproduces very accurately the full numerical result, for the region of our interest with large $m_{M} \sim 10^{14-15} \mathrm{GeV}$.

With respect to the numerical results, figure 2 exemplifies the main features of the extra Higgs mass corrections $\Delta m_{h}^{\mathrm{m} \overline{\mathrm{DR}}}$ due to neutrinos and sneutrino loops in terms of the two physical Majorana neutrino masses, $m_{N}$ and $m_{v}$. For values of $m_{N}<3 \times 10^{13} \mathrm{GeV}$ and $\left|m_{v}\right|<0.1-0.3 \mathrm{eV}$ the corrections to $M_{h}$ are positive and smaller than $0.1 \mathrm{GeV}$. In this region, the gauge contribution dominates. In fact, the wider black contour line with fixed $\Delta m_{h}^{\mathrm{m} \overline{\mathrm{DR}}}=0.09$ coincides with the prediction for the case where just the gauge part in the self-energies has been included. This means that 'the distance' of any other contour-line with respect to this one represents the difference in the radiative corrections respect to the MSSM prediction.

However, for larger values of $m_{N}$ and/or $\left|m_{v}\right|$ the Yukawa part dominates, and the radiative corrections become negative and larger in absolute value, up to values of $-5 \mathrm{GeV}$ in the right upper corner of Fig. 2. These corrections grow in modulus proportionally to $m_{M}$ and $m_{v}$, due to the fact that the seesaw mechanism imposes a relation between the three masses involved, $m_{D}^{2}=\left|m_{v}\right| m_{N}$.

Finally, we plot in Fig. 3, the contour-lines for fixed $\Delta m_{h}^{\mathrm{m} \overline{\mathrm{DR}}}$ in the less conservative case where $m_{\tilde{R}}$ is close to $m_{M}$. These are displayed as a function of $\left|m_{v}\right|$ and the ratio $m_{\tilde{R}} / m_{M} . m_{M}$ is fixed here to the reference value, $m_{M}=10^{14} \mathrm{GeV}$. For the interval studied here, we see again that the radiative corrections can be negative and as large as tens of $\mathrm{GeV}$ in the upper right corner of the plot. For instance, $\Delta m_{h}^{\mathrm{m} \overline{\mathrm{DR}}}=-30 \mathrm{GeV}$ for $m_{M}=10^{14} \mathrm{GeV},\left|m_{v}\right|=0.6 \mathrm{eV}$ and $m_{\tilde{R}} / m_{M}=0.7$. 


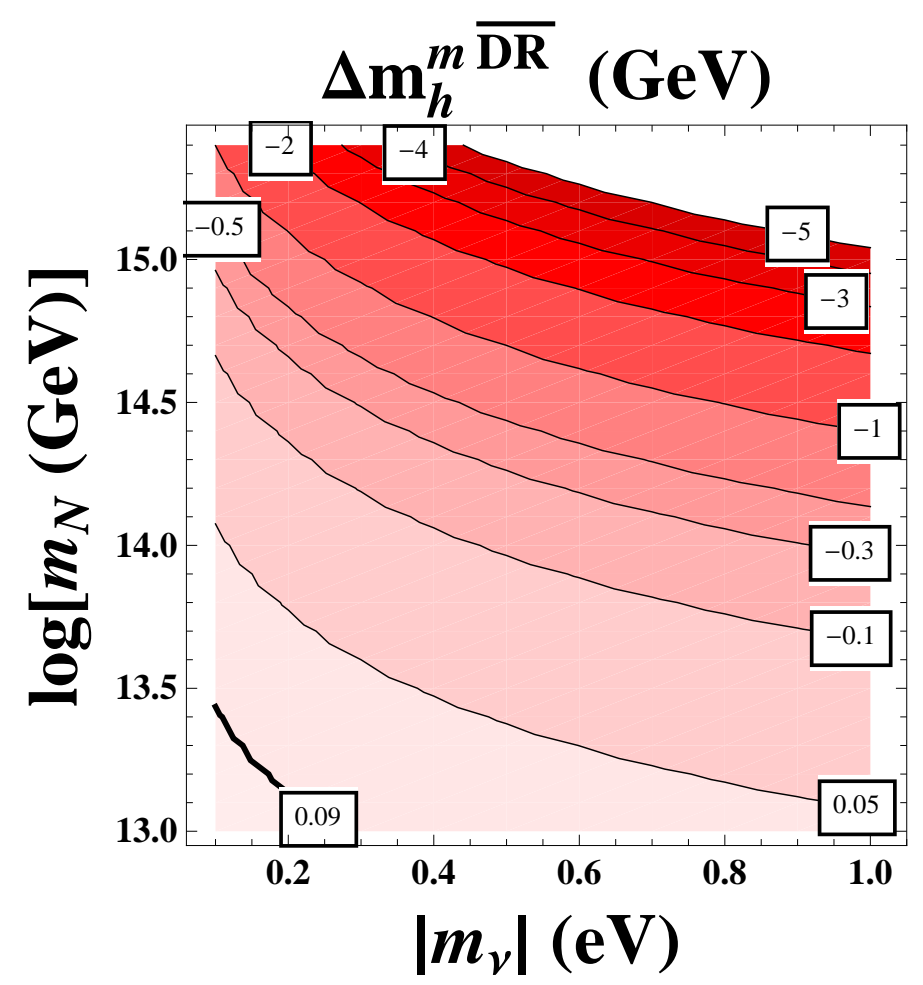

Figure 2: Contour-lines for the Higgs mass corrections from the neutrino/sneutrino sector as a function of the physical Majorana neutrino masses, light $\left|m_{V}\right|$ and heavy $m_{N}$. The other parameters are fixed to: $A_{v}=B_{V}=m_{\tilde{L}}=m_{\tilde{R}}=10^{3} \mathrm{GeV}, \tan \beta=5, M_{A}=200 \mathrm{GeV}$ and $\mu=200 \mathrm{GeV}$.

\section{Conclusions}

We have used the Feynman diagrammatic approach for the calculation of the radiative corrections to the lightest Higgs boson mass of the MSSM-seesaw. This method does not neglect the external momentum of the incoming and outgoing particles as it happens in the effective potential approach. We have performed a full calculation, obtaining not only the leading logarithmic terms as it would be the case in a RGE computation but also the finite terms, that we have seen that can be sizable for heavy Majorana neutrinos $\left(10^{13}-10^{15} \mathrm{GeV}\right)$ and the lightest neutrino mass within a range inspired by data $(0.1-1 \mathrm{eV})$. For some regions of the MSSM-seesaw parameter space, the corrections to $M_{h}$ are substantially larger than the anticipated LHC precision ( $\left.\sim 200 \mathrm{MeV}\right)$ [8]. Specifically they can be negative and up to $-5 \mathrm{GeV}$ if $m_{M} \sim 10^{15} \mathrm{GeV}$ and $\left|m_{v}\right| \sim 1 \mathrm{eV}$ and up to minus tens of $\mathrm{GeV}$ if in addition $m_{\tilde{R}}$ is also large and of similar size to $m_{M}$.

\section{Acknowledgements}

M. Herrero wishes to thank the organizors of RADCOR2011 for this fruitful and enjoyable meeting at the impresive site of Mamallapuram, India. 


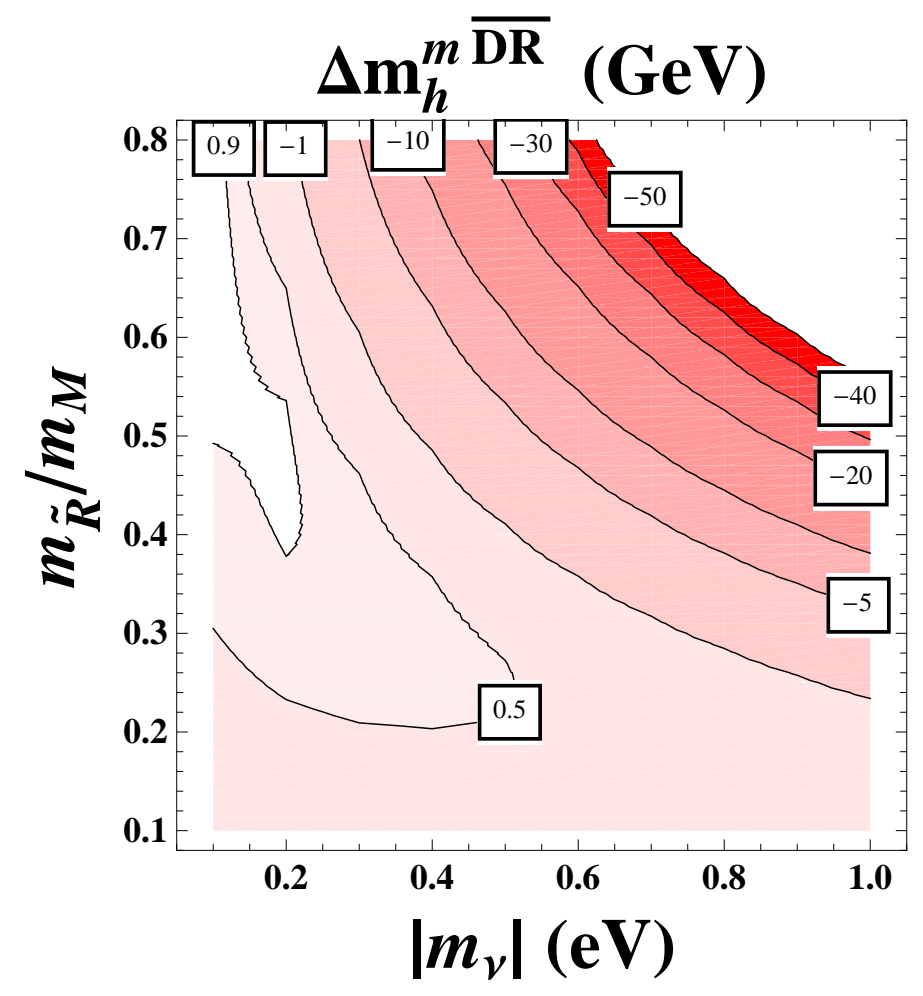

Figure 3: Contour-lines for the Higgs mass corrections from the neutrino/sneutrino sector as a function of the ratio $m_{\tilde{R}} / m_{M}$ and the lightest Majorana neutrino mass $\left|m_{V}\right|$. The other parameters are fixed to: $m_{M}=$ $10^{14} \mathrm{GeV}, A_{v}=B_{v}=m_{\tilde{L}}=10^{3} \mathrm{GeV}, \tan \beta=5, M_{A}=200 \mathrm{GeV}$ and $\mu=200 \mathrm{GeV}$

\section{References}

[1] P. Minkowski, Phys. Lett. B 67 (1977) 421;

[2] F. Borzumati and A. Masiero, Phys. Rev. Lett. 57 (1986) 961; M. Raidal et al., Eur. Phys. J. C 57, 13 (2008) [arXiv:0801.1826 [hep-ph]].

[3] S. Heinemeyer, M. J. Herrero, S. Penaranda and A. M. Rodriguez-Sanchez, JHEP 1105 (2011) 063 [arXiv:1007.5512 [hep-ph]].

[4] M. Frank, T. Hahn, S. Heinemeyer, W. Hollik, H. Rzehak and G. Weiglein, JHEP 0702 (2007) 047 [arXiv:0611326 [hep-ph]].

[5] J. Collins, F. Wilczek and A. Zee, Phys. Rev. D 18 (1978) 242.

[6] J. Küblbeck, M. Böhm and A. Denner, Comput. Phys. Commun. 60 (1990) 165;

T. Hahn, Comput. Phys. Commun. 140 (2001) 418 [arXiv:hep-ph/0012260];

T. Hahn and C. Schappacher, Comput. Phys. Commun. 143 (2002) 54 [arXiv:hep-ph/0105349].

The program and the user's guide are available via www. feynarts. de .

[7] T. Hahn and M. Pérez-Victoria, Comput. Phys. Commun. 118 (1999) 153 [arXiv:hep-ph/9807565].

[8] G. Aad et al. [The ATLAS Collaboration], arXiv:0901.0512;

G. Bayatian et al. [CMS Collaboration], J. Phys. G 34 (2007) 995. 